\title{
Synergistic Activity of Colistin Combined With Auranofin Against Colistin-Resistant Gram-Negative Bacteria
}

\author{
Xiaoxuan Feng 1,2, Shuai Liu'2,3, Yang Wang ${ }^{2,3}$, Yulin Zhang ${ }^{2,3,4}$, Lingxiao Sun ${ }^{2,3}$, \\ Haibo Li ${ }^{2,3,4}$, Chunlei Wang ${ }^{2,3,4}$, Yingmei Liu, ${ }^{2,3,4 *}$ and Bin Cao ${ }^{1,2,3,4,5 *}$ \\ 1 Graduate School of Peking Union Medical College, Chinese Academy of Medical Sciences, Peking Union Medical College, \\ Beijing, China, ${ }^{2}$ Department of Pulmonary and Critical Care Medicine, Center for Respiratory Diseases, China-Japan \\ Friendship Hospital, Beijing, China, ${ }^{3}$ China-Japan Friendship Hospital, National Clinical Research Center for Respiratory \\ Diseases, Clinical Center for Pulmonary Infections, Capital Medical University, Beijing, China, ${ }^{4}$ Laboratory of Clinical \\ Microbiology and Infectious Diseases, Department of Pulmonary and Critical Care Medicine, China-Japan Friendship \\ Hospital, Beijing, China, ${ }^{5}$ Tsinghua University-Peking University Joint Center for Life Sciences, Tsinghua University, Beijing, \\ China
}

\section{OPEN ACCESS}

Edited by:

Haruyoshi Tomita,

Gunma University, Japan

Reviewed by:

Emanuele Durante Mangoni,

University of Campania Luigi Vanvitelli,

Younes Smani

Institute of Biomedicine of Seville

(IBIS), Spain

*Correspondence:

Yingmei Liu

lym13601063223@126.com

Bin Cao

caobin_ben@163.com

Specialty section:

This article was submitted to

Antimicrobials, Resistance

and Chemotherapy,

a section of the journal

Frontiers in Microbiology

Received: 05 March 2021

Accepted: 03 June 2021

Published: 25 June 2021

Citation:

Feng $X$, Liu S, Wang $Y$, Zhang $Y$,

Sun L, Li H, Wang C, Liu Y and Cao B

(2021) Synergistic Activity of Colistin

Combined With Auranofin Against

Colistin-Resistant Gram-Negative

Bacteria. Front. Microbiol. 12:676414.

doi: 10.3389/fmicb.2021.676414
Colistin-resistant (Col-R) bacteria are steadily increasing, and are extremely difficult to treat. New drugs or therapies are urgently needed to treat infections caused by these pathogens. Combination therapy with colistin and other old drugs, is an important way to restore the activity of colistin. This study aimed to investigate the activity of colistin in combination with the anti-rheumatic drug auranofin against Col-R Gramnegative bacteria. The results of checkerboard analysis demonstrated that auranofin synergized with colistin against Col-R Gram-negative bacteria. Time-kill assays showed significant synergistic antimicrobial activity of colistin combined with auranofin. Electron microscopy revealed that the combination resulted in more cellular structural alterations compared to each drug alone. Auranofin enhanced the therapeutic effectiveness of colistin in mouse peritoneal infection models. These results suggested that the combination of colistin and auranofin might be a potential alternative for the treatment of Col-R Gram-negative bacterial infections.

Keywords: colistin, auranofin, colistin-resistant, repurposing, combination therapy, synergistic effect

\section{INTRODUCTION}

Polymyxins (including polymyxin B and colistin) are considered as last resort drugs for the treatment of infections caused by extensively drug-resistant Gram-negative bacteria. Polymyxins act on the outer membrane of Gram-negative bacteria, disrupt the stability of cell membrane, cause osmotic imbalance, and ultimately lead to cell death (Velkov et al., 2010; Berglund et al., 2015). Before 2015, mutations in chromosomal genes, such as $p m r A B$, $p h o P Q$, and $m g r B$, were considered as the main causes of high-level polymyxin resistance (Olaitan et al., 2014). However, the discovery of plasmid-encoded mcr-1 gene led to global reports of polymyxin resistance (Liu et al., 2016; Wang R. et al., 2018).

The gradual emergence of the polymyxin-resistant bacterial strains has greatly limited the antibiotic therapy options. It has been shown that repurposing FDA-approved non-antibiotic drugs 
in combination with polymyxin could be a promising alternative therapeutic strategy (Schneider et al., 2016; Ayerbe-Algaba et al., 2018, 2019; Cannatelli et al., 2018; Tran et al., 2018; Wang Y.M. et al., 2018; Zhou et al., 2018; Falagas et al., 2019; Hussein et al., 2020). To date, a number of FDA-approved non-antibiotic drugs, e.g., azidothymidine (Falagas et al., 2019), oxyclozanide (Ayerbe-Algaba et al., 2019), niclosamide (AyerbeAlgaba et al., 2018), resveratrol (Cannatelli et al., 2018), mitotane (Tran et al., 2018), sertraline (Hussein et al., 2020), ivacaftor (Schneider et al., 2016), eugenol (Wang Y.M. et al., 2018), pterostilbene (Zhou et al., 2018), in combination with polymyxins have displayed synergistic killing against polymyxin-resistant Gram-negative bacteria. Auranofin is an FDA-approved antirheumatoid arthritis drug (Roder and Thomson, 2015). Adverse effects are associated with the long-term (months to years) use of auranofin, including diarrhea (40\% of subjects), skin rashes (2-5\%), hematologic abnormalities (rare), and proteinuria (5\%; Kean et al., 1997). However, the most common side effect, diarrhea, can be easily managed (Suarez-Almazor et al., 2000). Hence, its largely acceptable toxicity paves the way to its repositioning for new and different therapeutic uses. Auranofin exerts a significant antimicrobial activity against numerous Gram-positive bacteria (Aguinagalde et al., 2015; Harbut et al., 2015; Tharmalingam et al., 2019), which is purported to inhibit the thioredoxin reductase ( $\operatorname{TrxR}$ ) of Gram-positive bacteria and disrupt the redox balance, resulting in cell death. However, auranofin alone has limited activity against Gram-negative bacteria (Harbut et al., 2015). Some studies have shown that colistin in combination with auranofin is effective against MDR Gram-negative bacteria (Sun et al., 2016; Torres et al., 2018). A recent study showed that auranofin could restore the activity of colistin against several $m c r-1$ positive Enterobacteriaceae (Sun et al., 2020). However, it is not known if auranofin can restore the activity of colistin against Colistin-resistant (Col-R) Gram-negative bacteria of different species with different colistin resistance mechanisms. In this study, the in vitro activities of colistin in combination with auranofin were evaluated against a group of clinical Col-R Gram-negative bacteria including Klebsiella pneumoniae, Escherichia coli, Pseudomonas aeruginosa, and Acinetobacter baumannii. In addition, we explored the therapeutic effectiveness of colistin combined with auranofin in mouse peritoneal infection models.

\section{MATERIALS AND METHODS}

\section{Bacterial Isolates and Chemicals}

We used 23 clinical Col-R isolates in this study. Four reference colistin-susceptible (Col-S) K. pneumoniae ATCC 700603, E. coli ATCC25922, $P$. aeruginosa ATCC27853, and A. baumannii ATCC19606 isolates were also used. Colistin and auranofin were purchased from the National Institutes for Food and Drug Control (Beijing, China). Colistin solutions were prepared in sterile Milli-Q water before the experiments. Stock solutions of auranofin were prepared in dimethyl sulfoxide (DMSO) and then diluted with sterile Milli-Q water to ensure a final DMSO concentration of $\leq 5 \%$ (v/v; Lin et al., 2018).

\section{In vitro Susceptibility Testing and Colistin Resistance Mechanisms}

The minimum inhibitory concentrations (MICs) of colistin and auranofin were determined by the method described in the Clinical and Laboratory Standards Institute, 2019). Colistin or auranofin was prepared with two-fold serial dilutions. A final bacterial suspension at $5 \times 10^{5} \mathrm{CFU} / \mathrm{mL}$ was added in each well, and incubated with two-fold serial dilutions of colistin or auranofin for $18 \mathrm{~h}$ at $37^{\circ} \mathrm{C}$. MIC was determined as the lowest concentration that inhibited the visible growth of the bacteria. Colistin resistance mechanisms were analyzed with detection of pmrA, pmrB, phoP, phoQ, mgr $B, m c r-1$, and $m c r-8$ genes by PCR (Liu et al., 2016; Wang X. et al., 2018; Ayerbe-Algaba et al., 2019). The sequences of the PCR products were determined by RuiBiotech (Beijing, China).

\section{Checkerboard Assays}

The synergistic interaction between colistin and auranofin was tested using the checkerboard technique (Flamm et al., 2019). Colistin and auranofin were prepared with two-fold serial dilutions and mixed to create different concentration combinations. A final bacterial suspension at $5 \times 10^{5} \mathrm{CFU} / \mathrm{mL}$ was added in each well. After incubation for $18 \mathrm{~h}$ at $37^{\circ} \mathrm{C}$, the optimal fractional inhibitory concentration index (FICI) was calculated as the MIC of the combination divided by that of each compound used alone. FICI $\leq 0.5$ denotes synergy, FICI $>0.5-$ 4 denotes no interaction and FICI $>4$ denotes antagonism (Odds, 2003).

\section{Time-Kill Assays}

Time-kill assays were performed to further evaluate the synergistic effect of colistin with auranofin according to Hu's method (Hu et al., 2019) with minor modifications. The concentration of colistin was used at 2 or $4 \mathrm{mg} / \mathrm{L}$. The concentrations of auranofin were chosen according to the concentrations that showed synergistic effect with colistin in the checkerboard assays. Fresh cultures were mixed with colistin or auranofin alone or a combination and incubated at $37^{\circ} \mathrm{C}$ with shaking. Thereafter, $10 \mu \mathrm{L}$ of each suspension was plated on nutrient agar plates for viable bacterial quantification after serial dilution at different time points of incubation. Synergistic activity was defined as a $\geq 2 \log _{10}$ decrease in CFU/mL of the combination compared to the most active monotherapy (Doern, 2014).

\section{Scanning Electron and Transmission Electron Microscopy}

The impact of colistin in combination with auranofin on the cellular morphology of the high-level Col-R K. pneumoniae 18605 and A. baumannii 13660 was examined by scanning electron microscopy (SEM) and transmission electron microscopy (TEM). For K. pneumoniae 18605, a log phase culture was treated with $2 \mathrm{mg} / \mathrm{L}$ colistin, $2 \mathrm{mg} / \mathrm{L}$ auranofin, or both for $2 \mathrm{~h}$ in cationadjusted Mueller-Hinton broth (CAMHB). For A. baumannii 13660, a $\log$ phase culture was treated with $2 \mathrm{mg} / \mathrm{L}$ colistin, $4 \mathrm{mg} / \mathrm{L}$ auranofin, or both for $2 \mathrm{~h}$ in CAMHB. Bacterial pellets were obtained by centrifugation at $4,000 \mathrm{~g}$ for $10 \mathrm{~min}$ twice. 
Next, the pellets were fixed overnight at $4^{\circ} \mathrm{C}$ with $1 \mathrm{~mL} 2.5 \%$ glutaraldehyde. The fixatives were removed after centrifugation at $4,000 \mathrm{~g}$ for $10 \mathrm{~min}$, and finally the bacterial pellets were resuspended in $1 \mathrm{~mL}$ PBS. SEM was performed with a HITACHI SU8020 scanning electron microscope. For TEM observation, all images were acquired using a JEM-1200EX microscope.

\section{In vivo Treatment Evaluation}

To further evaluate the in vivo effect of colistin in combination with auranofin, two infection models were established in female ICR mice. 6-8 weeks old mice (weighing an average of $20 \mathrm{~g}$ ) were purchased from Beijing Vital River Laboratory Animal Technology Co. Ltd. For the bacterial load experiment, A dose of $2 \times 10^{6}$ CFU K. pneumoniae 18605 bacterial suspension was intraperitoneally (i.p.) injected into the mice. After $1 \mathrm{~h}$ of infection, mice were i.p. treated with vehicle, colistin sulfate $(1 \mathrm{mg} / \mathrm{kg})$ or auranofin $(0.5 \mathrm{mg} / \mathrm{kg})$ alone or their combination ( $n=8$ per group). All mice were euthanized at $14 \mathrm{~h}$ post-infection. Peritoneal fluid (PF) was collected by injecting $2 \mathrm{~mL}$ sterile saline solution into the peritoneum, followed by gentle massage and aspiration. The spleen was aseptically obtained, weighed and homogenized. Individual samples were serially diluted in sterile saline solution and $0.1 \mathrm{~mL}$ aliquots were placed on nutrient agar plates. The colonies were counted after incubation overnight at $37^{\circ} \mathrm{C}$. The bacterial loads in PF and spleen between groups were compared using one-way ANOVA and the post hoc Bonferroni test. A $p$-value $<0.05$ was considered significant.

For survival assay, mice were infected i.p. with $1 \times 10^{7} \mathrm{CFU}$ of A. baumannii 13660. After $0.5 \mathrm{~h}$ of infection, mice $(n=8$ per group) were i.p. treated with vehicle or colistin sulfate $(1.5 \mathrm{mg} / \mathrm{kg})$ or auranofin $(0.5 \mathrm{mg} / \mathrm{kg})$ alone or in combination. The same treatment procedure was then repeated once daily until the end of the study. Survival rates were monitored for 5 days. Log-rank (Mantel-Cox) test was used to compared the survival distributions of different groups. A $p$-value $<0.05$ was considered significant.

\section{Ethics Statement}

The animal experiments were performed in accordance with the national standards for laboratory animals in China, with approval being provided by the Animal Ethical and Welfare Committee of the China-Japan Friendship Hospital (Zryhyy11-20-07-1).

\section{RESULTS}

\section{Synergistic Effect of Colistin and Auranofin in Checkerboard Assays}

Minimum inhibitory concentrations of colistin and auranofin were tested against a group of 27 strains of four different Gram-negative bacterial species. As shown in Table 1, colistin MICs of the Col-R strains ranged from 4 to $1,024 \mathrm{mg} / \mathrm{L}$, while those for the Col-S reference strains were $0.5-1 \mathrm{mg} / \mathrm{L}$. MICs of auranofin were between 8 and $512 \mathrm{mg} / \mathrm{L}$ for all test strains. Mechanisms of resistance to colistin were identified in some of the strains studied (Table 1). Checkerboard assays revealed that the combination of colistin and auranofin showed a significant synergistic effect $(\mathrm{FICI} \leq 0.5)$ against Col-R strains with different colistin resistance mechanisms. Additionally, the colistin MICs of most Col-R strains in the presence of auranofin were reduced to $\leq 2 \mathrm{mg} / \mathrm{L}$. For all the Col-S reference strains, no interaction effect (FICI > 0.5) was observed.

\section{Time-Kill Assays of Colistin in Combination With Auranofin Against Col-R Strains}

The synergistic effect of colistin in combination with auranofin was tested using time-kill assays against seven selected Col$\mathrm{R}$ strains, namely $m \mathrm{cr}-8$ positive $K$. pneumoniae C505, mcr- 1 positive K. pneumoniae $09-20$, $m c r-1$ positive E. coli $08-85$, highlevel Col-R K. pneumoniae 18605, E. coli C1157, P. aeruginosa 26587, and A. baumannii 13660. As shown in Figure 1, for all three $m c r$ gene positive isolates, neither colistin nor auranofin monotherapy showed any bacterial killing effect, and the growth curves were similar to that of control. The combination of colistin and auranofin resulted in the elimination of all three $m c r$ gene positive isolates at $4 \mathrm{~h}$, with no regrowth observed for K. pneumoniae C505 and K. pneumoniae 09-20. Interestingly, regrowth was observed for E. coli $08-85$ after $8 \mathrm{~h}$ of treatment. As shown in Figure 2, for all the high-level Col-R isolates, auranofin monotherapy displayed no antimicrobial activity. Similar to auranofin, colistin monotherapy also could not prevent the growth of all high-level Col-R strains except $K$. pneumoniae 18605 , wherein colistin monotherapy reduced the initial bacterial count slightly in the first $2 \mathrm{~h}$, however, regrowth to the control value was observed at $24 \mathrm{~h}$. When colistin was combined with auranofin, no viable bacterial cells were detected within 2-24 h for all high-level Col-R strains.

\section{Impact of Colistin and Auranofin on Cellular Morphology}

We performed SEM and TEM experiments to determine the morphological changes of $K$. pneumoniae 18605 and A. baumannii 13660 induced by colistin, auranofin, or both. For K. pneumoniae 18605, SEM images showed that auranofin monotherapy displayed no morphological changes (Figure 3C) compared to the control group (Figure 3A). Membrane blebbing was evidently observed with colistin monotherapy (Figure 3B). The combination treatment caused large-scale membrane disruptions (Figure 3D). By TEM, treatment with auranofin monotherapy did not show any impact on the cellular morphology (Figure 3G) compared to the control group (Figure 3E). However, minor protrusions were caused by colistin monotherapy (Figure 3F). With the combination, bacterial cell surface was extensively disrupted and showed cell lysis (Figure $3 \mathbf{H}$ ). For A. baumannii 13660, SEM images showed that colistin (Supplementary Figure 1B) and auranofin (Supplementary Figure 1C) monotherapy displayed no morphological changes compared to the control group (Supplementary Figure 1A). The combination treatment (Supplementary Figure 1D) resulted in a significant reduction 
TABLE 1 | Checkerboard assays showing the effects of colistin combined with auranofin.

\begin{tabular}{|c|c|c|c|c|c|c|c|c|}
\hline Pathogen & Strains & Source & Mechanism of colistin resistance & \multicolumn{4}{|c|}{ MIC(mg/L) } & $\mathrm{FICl}$ \\
\hline & 12959 & BALF & PmrA G53V & 64 & 128 & 0.5 & 4 & 0.039 \\
\hline & 15979 & Sputum & ISKpn14 at nt115 of mgrB & 64 & 256 & 1 & 4 & 0.031 \\
\hline & 13649 & BALF & ISKpn14 at nt126 of mgrB & 128 & 256 & 2 & 2 & 0.023 \\
\hline & 18229 & BALF & PhoQ A63E & 256 & 512 & 1 & 4 & 0.012 \\
\hline & C505 & Sputum & $m c r-8$ & 8 & 128 & 0.5 & 2 & 0.078 \\
\hline & C270 & Urine & $m c r-8$ & 16 & 128 & 1 & 1 & 0.07 \\
\hline & 17-R27 & Liver & $m c r-1$ & 32 & 256 & 1 & 4 & 0.047 \\
\hline & $09-20$ & Blood & $m c r-1$ & 16 & 256 & 0.5 & 2 & 0.039 \\
\hline & C1157 & Sputum & PhoQ V413F & 64 & 64 & 1 & 1 & 0.031 \\
\hline & C1279 & Urine & PmrA S29G & 64 & 64 & 0.5 & 1 & 0.023 \\
\hline & C1461 & Urine & PmrB Y358M, PhoP I44L & 32 & 64 & 0.5 & 2 & 0.047 \\
\hline & 17-R14 & Liver & $m c r-1$ & 4 & 8 & 0.25 & 2 & 0.313 \\
\hline & $08-85$ & Blood & $m c r-1$ & 8 & 16 & 1 & 1 & 0.188 \\
\hline \multirow[t]{4}{*}{$P$. aeruginosa } & ATCC27853 & ATCC & Colistin susceptible & 1 & 512 & 1 & 2 & 1.004 \\
\hline & 26751 & BALF & PhoP V99L & 128 & 512 & 4 & 4 & 0.039 \\
\hline & 26587 & BALF & PhoP V99L & 512 & 512 & 2 & 8 & 0.02 \\
\hline & 26683 & BALF & ND & 64 & 512 & 4 & 0.5 & 0.063 \\
\hline \multirow[t]{2}{*}{ A. baumannii } & ATCC19606 & ATCC & Colistin susceptible & 1 & 16 & 0.5 & 2 & 0.625 \\
\hline & 13660 & Sputum & PmrA T119s & 512 & 32 & 2 & 4 & 0.129 \\
\hline
\end{tabular}

BALF, bronchoalveolar lavage fluid; Col, colistin; Aur, auranofin; FICl, fractional inhibitory concentration index; and ND, not determined.
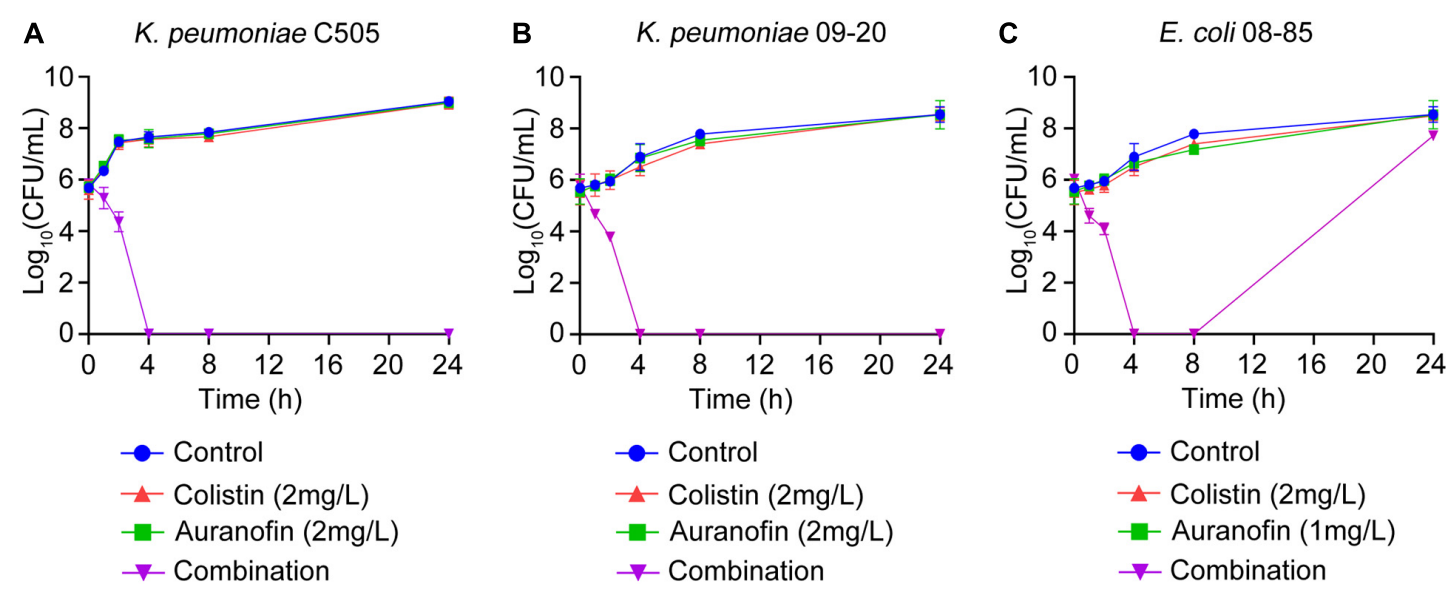

FIGURE 1 | Time-kill assays showing the effects of colistin or auranofin alone or in combination against $m c r-8$ positive $K$. pneumoniae C505 (A), mcr-1 positive K. pneumoniae 09-20 (B), and mcr-1 positive E. coli 08-85 (C).

in cell length, and the cell surface was more uneven compared to the control group (Supplementary Figure 1A). For TEM, auranofin monotherapy (Supplementary Figure 1G) did not affected the cell surface. Membrane blebbing was observed with colistin monotherapy (Supplementary Figure 1F). Bacterial cells treated with the combination (Supplementary Figure 1H) were much shorter in length compared to the control group (Supplementary Figure 1E). 


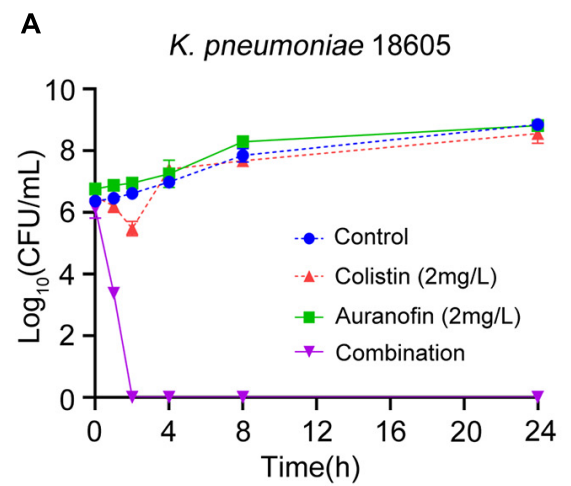

C

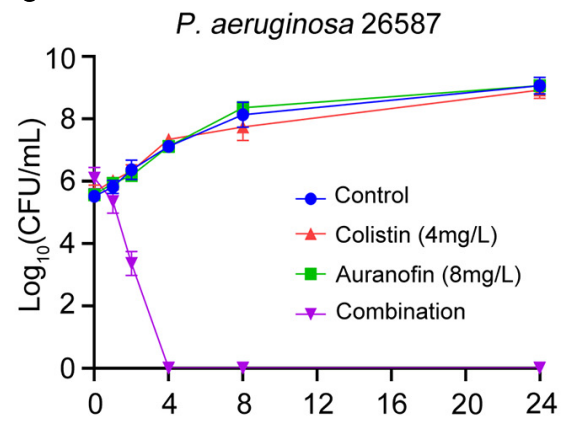

B

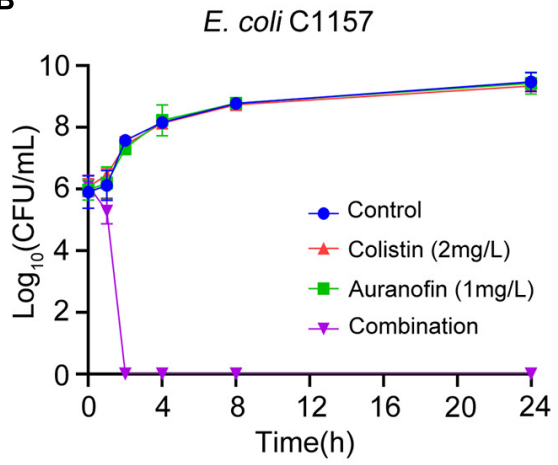

D

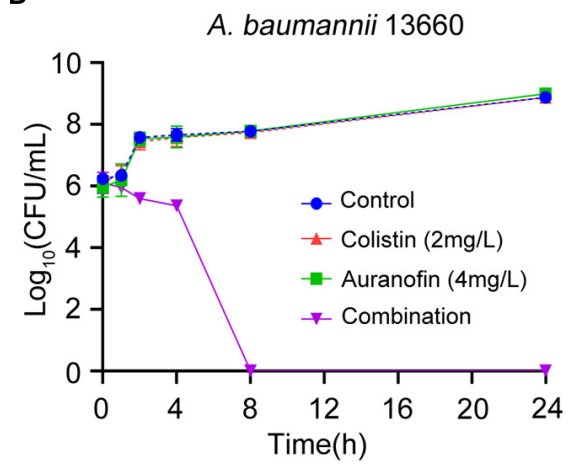

FIGURE 2 | Time-kill assays showing the effects of colistin or auranofin alone or in combination against high-level Col-R K. pneumoniae 18605 (A), E. coli C1157 (B), P. aeruginosa 26587 (C), and A. baumannii 13660 (D).
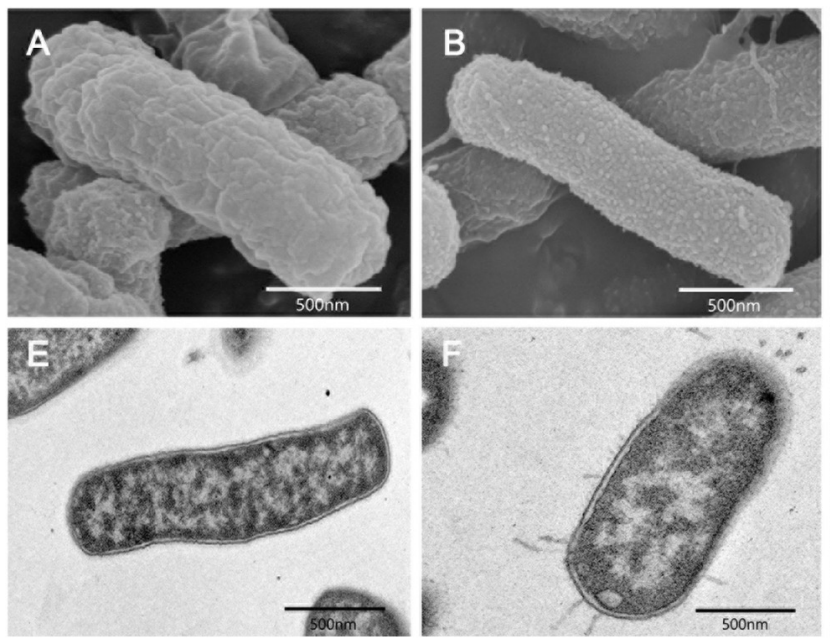
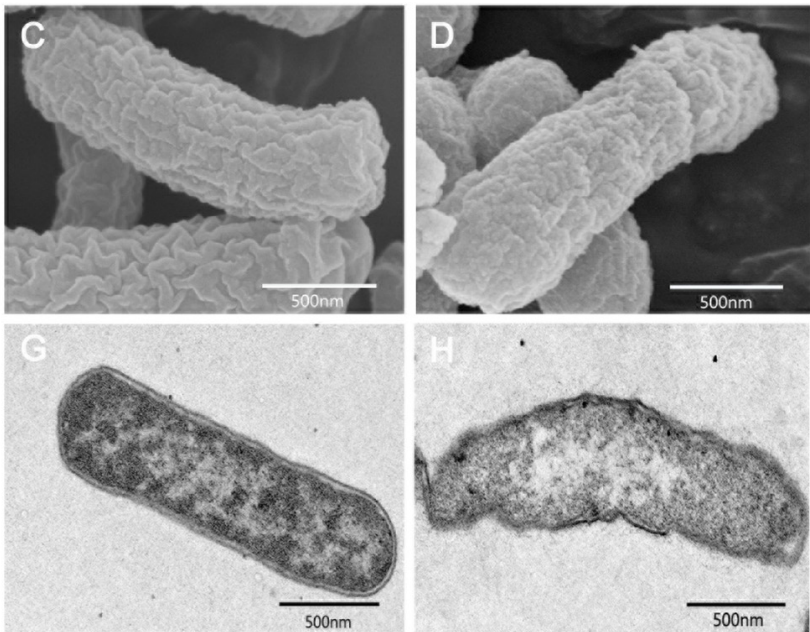

FIGURE 3 | SEM and TEM images of high-level Col-R K. pneumoniae 18605 after treatment with 2 mg/L colistin alone (B,F), 2 mg/L auranofin alone (C,G), or combination (D,H) for 2 h. (A,E) represent the control condition.

\section{Efficacy of Combination Therapy in vivo}

The in vivo efficacy of colistin in combination with auranofin was tested using two murine models of K. pneumoniae 18605 and A. baumannii 13660 infection. The dose of auranofin was selected based on the in vitro synergistic potentiation (Table $\mathbf{1}$ and Figures 1, 2), and was much lower than the maximum tolerated dose (Harbut et al., 2015). Colistin sulfate was dosed at approximately the human equivalent dose ( $\mathrm{Li}$ et al., 2018). As shown in Figure 4, we evaluated the bacterial loads in the PF and spleen of mice. Neither colistin nor auranofin monotherapy showed antimicrobial activity against the infected bacteria (Figures $4 \mathbf{A}, \mathbf{B}$ ). In contrast, the combination of colistin 


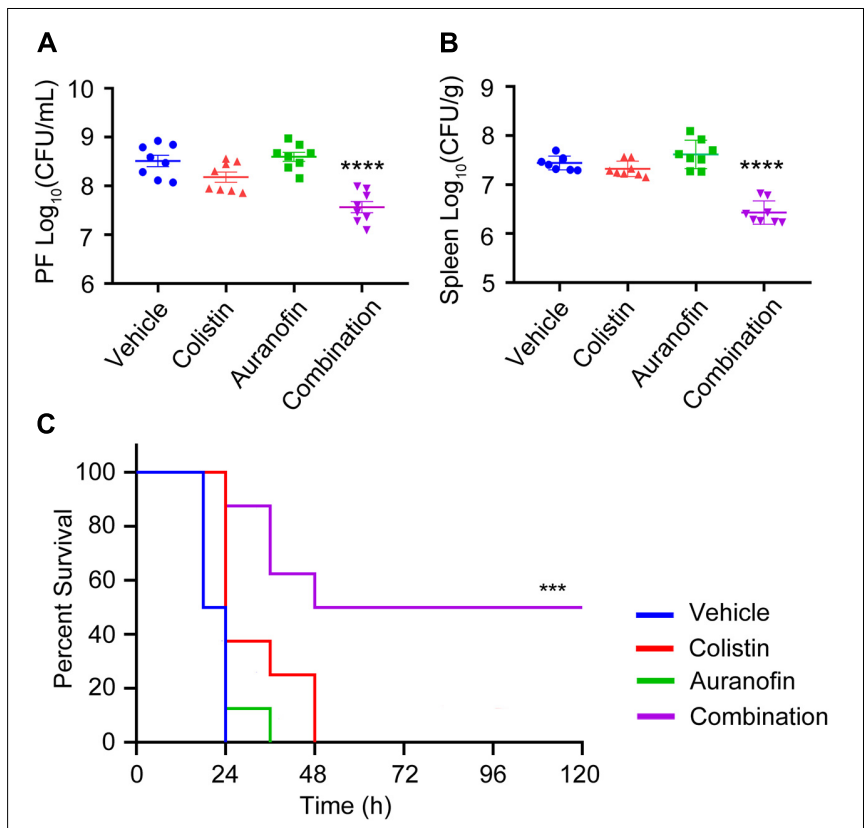

FIGURE 4 | The combination of colistin and auranofin shows potency in vivo. (A,B) Mice were infected by high-level Col-R K. pneumoniae 18605 and received single dose of i.p. administration of vehicle, colistin sulfate, auranofin, or their combination ( $n=8$ per group). Bacterial loads in the PF (A) and spleen (B) are shown. (C) Survival curves showing combination efficacies in the peritoneal infection model. Mice were infected by a lethal dose of high-level Col-R A. baumannii 13660 and treated with one dose at $0.5 \mathrm{~h}$ post infection, followed by once-daily treatment with i.p. vehicle, colistin sulfate, auranofin, or the combination ( $n=8$ per group). ${ }^{* \star \star *}$ indicates $p \leq 0.0001$, ${ }^{\star \star \star}$ indicates $p \leq 0.001$, compared with the control group.

and auranofin proved efficacious, with almost 10 -fold bacterial load reduction $(p<0.0001)$ in the PF and spleen than that of the control group (Figures 4A,B). In the A. baumannii 13660 infection mouse model, monotherapy treatments administered $0.5 \mathrm{~h}$ post-infection did not demonstrate any significant survival beyond that of the control group (Figure 4C). However, animals receiving colistin and auranofin combination therapy daily for 5 days could rescue $50 \%$ of those treated (Figure 4C).

\section{DISCUSSION}

As a drug of last resort, colistin was used to treat infections caused by MDR Gram-negative bacteria. However, with the consumption of colistin, the number of Col-R strains increased (Bialvaei and Samadi Kafil, 2015). Since the discovery of $m c r-1$ plasmid and its spread worldwide (Liu et al., 2016; Wang R. et al., 2018), the problem of colistin resistance has aroused widespread concern. Hence, it is important to restore the activity of colistin through combination therapy. In this study, we evaluated the potential application of colistin combined with auranofin to treat infections caused by Col-R isolates.

The in vitro results showed that auranofin exhibited significant synergy with colistin against Col-R Gram-negative bacteria including $m c r$ positive strains. Similarly, a recent study reported that auranofin restored the activity of colistin against bacteria carrying $m c r-1$ gene or its variants/homologs (Sun et al., 2020). Two previous studies reported that with the addition of auranofin, the effects of colistin, and auranofin against MDR Gram-negative bacteria were significantly increased (Sun et al., 2016; Torres et al., 2018).

In vivo study showed that colistin combined with auranofin improved the mice survival rate, and reduced the bacterial loads of K. pneumoniae 18605 in the PF and spleen of mice (Figures 4A,B). In vitro study on K. pneumoniae 18605 strain showed that the combined application of both colistin and auranofin at $2 \mathrm{mg} / \mathrm{L}$ dose could significantly reduce the initial bacterial count to the limit of detection (Figure 2A). However, the reduction of bacterial loads in vivo was much less than that in in vitro study. This discrepancy might be related to the pharmacokinetic properties of colistin and auranofin. The peak plasma concentration of colistin in mice treated with a single dose of colistin sulfate $(2.5 \mathrm{mg} / \mathrm{kg}$ ) was $3.08 \mathrm{mg} / \mathrm{L}$ (Li et al., 2018). Capparelli et al. (2017) have found that oral administration of $6 \mathrm{mg}$ of auranofin daily for 7 days could result in a mean $C_{\max }$ value of $0.312 \mathrm{mg} / \mathrm{L}$ in plasma, which is far below its in vitro concentration of $2 \mathrm{mg} / \mathrm{L}$ used in this study. Using Monte-Carlo simulations, it has been shown that daily oral administration of $21 \mathrm{mg}$ of auranofin could result in $C_{\max }$ of auranofin's plasma concentration ranging between 0.4 and $1.6 \mathrm{mg} / \mathrm{L}$ (Capparelli et al., 2017). When mice were intraperitoneally administered with a single dose of $1 \mathrm{mg} / \mathrm{kg}$ colistin sulfate and $0.5 \mathrm{mg} / \mathrm{kg}$ auranofin, the plasma concentration of each compound might not reach the corresponding in vitro concentration. Therefore, the combination therapy showed less antimicrobial activity in vivo than in vitro. The safety of auranofin in clinical use is well established. Auranofin has been extensively used in clinical setting at the FDA-approved human dose ( $6 \mathrm{mg} /$ day $)$, a steadystate blood gold concentration of $3.5 \mu \mathrm{M}$ would be reached in 12 weeks (Debnath et al., 2012). Patients with rheumatoid arthritis who were treated with auranofin ( $6 \mathrm{mg} /$ day $)$, had shown a safe toxicity profile (Suarez-Almazor et al., 2000). Patients with relapsed chronic lymphocytic leukemia who received 9 and $12 \mathrm{mg}$ of auranofin daily for at least 28 days, followed by up to $21 \mathrm{mg} /$ day, were well-tolerated (Clinical Trails registration no. NCT01419691). Given that the course of antimicrobial therapy is much shorter than that of treatment for rheumatoid arthritis or chronic lymphocytic leukemia, auranofin could be safely used to treat bacterial infections.

Scanning electron microscopy and TEM of K. pneumoniae 18605 and A. baumannii 13660 cells revealed that auranofin alone had no influence on cellular morphology (Figures 3C,G and Supplementary Figures 1C,G). Membrane blebbing was evidently observed when the cells were treated with colistin alone (Figure 3B and Supplementary Figure 1F). Similar changes have been observed in E. coli (Koike et al., 1969), $P$. aeruginosa (Hussein et al., 2020) after treatment with colistin alone. The bacterial outer membrane of K. pneumoniae 18605 was dramatically disrupted by the combination of colistin with auranofin, and showed cell lysis (Figures 3D,H). For A. baumannii 13660, the combination affected the overall structure of the strain, leading to an extensive shortening in the 
length of the bacteria (Supplementary Figures 1D,H). These observations suggested that auranofin may directly enhance the structural alteration effects of colistin.

Auranofin directly inhibited the TrxR in $S$. aureus and M. tuberculosis, leading to disruption of thiol-redox homeostasis and cell death (Harbut et al., 2015). However, the presence of glutathione system in several Gram-negative bacteria could maintain the redox balance and render auranofin ineffective (Harbut et al., 2015). A previous study has reported that the permeability barrier of outer membrane is the reason for the lack of activity of auranofin against Gram-negative bacteria (Thangamani et al., 2016). For the $m c r-1$ positive bacteria, auranofin was reported that irreversibly inhibited the function of MCR-1 via displacement of $\mathrm{Zn}$ (II) cofactors from the active site, thereby resensitizing $m c r-1$ positive bacteria to colistin (Sun et al., 2020). MCR-1 is a phosphoethanolamine (PEA) transferase, which confers colistin resistance by adding the PEA moiety to lipid A (Wang Y. et al., 2017). Further research is needed to identify the exact mode of action of auranofin when combined with colistin against high-lever Col$\mathrm{R}$ pathogens.

In summary, auranofin enhanced the in vitro and in vivo antimicrobial activities of colistin against Col-R Gram-negative bacteria. As auranofin is currently available, it can be easily used for antimicrobial therapy instead of developing new antimicrobial drugs. There is great potential for this novel combination to treat infections caused by Col-R Gramnegative bacteria.

\section{DATA AVAILABILITY STATEMENT}

The original contributions presented in the study are included in the article/Supplementary Material; further inquiries can be directed to the corresponding author/s.

\section{REFERENCES}

Aguinagalde, L., Díez-Martínez, R., Yuste, J., Royo, I., Gil, C., Lasa, I., et al. (2015). Auranofin efficacy against MDR Streptococcus pneumoniae and Staphylococcus aureus infections. J. Antimicrob. Chemother. 70, 2608-2617. doi: $10.1093 / \mathrm{jac} / \mathrm{dkv} 163$

Ayerbe-Algaba, R., Gil-Marqués, M. L., Jiménez-Mejías, M. E., Sánchez-Encinales, V., Parra-Millán, R., Pachón-Ibáñez, M. E., et al. (2018). Synergistic Activity of Niclosamide in Combination With Colistin Against Colistin-Susceptible and Colistin-Resistant Acinetobacter baumannii and Klebsiella pneumoniae. Front. Cell. Infect. Microb. 8:348. doi: 10.3389/fcimb.2018.00348

Ayerbe-Algaba, R., Gil-Marqués, M. L., Miró-Canturri, A., Parra-Millán, R., Pachón-Ibáñez, M. E., Jiménez-Mejías, M. E., et al. (2019). The anthelmintic oxyclozanide restores the activity of colistin against colistin-resistant Gramnegative bacilli. Internat. J. Antimicrob. Agents 54, 507-512. doi: 10.1016/j. ijantimicag.2019.07.006

Berglund, N. A., Piggot, T. J., Jefferies, D., Sessions, R. B., Bond, P. J., and Khalid, S. (2015). Interaction of the antimicrobial peptide polymyxin B1 with both membranes of E. coli: a molecular dynamics study. PLoS Computat. Biol. 11:e1004180. doi: 10.1371/journal.pcbi.1004180

Bialvaei, A. Z., and Samadi Kafil, H. (2015). Colistin, mechanisms and prevalence of resistance. Curr. Med. Res. Opin. 31, 707-721. doi: 10.1185/03007995.2015. 1018989

\section{ETHICS STATEMENT}

The animal study was reviewed and approved by Animal Ethical and Welfare Committee of the China-Japan Friendship Hospital (Zryhyy11-20-07-1).

\section{AUTHOR CONTRIBUTIONS}

BC and YL conceived and designed the study. XF, SL, YW, YZ, LS, HL, and CW carried out the experiments. XF performed the statistical analysis and wrote the draft. All authors read and approved the final version of the manuscript.

\section{FUNDING}

This work was funded by the Natural Science Foundation of China (No. 81970010/H0104), National Key Research and Development Program of China (2018YFC1200102), the Ministry of Science and Technology of China (2017ZX10103004), and the CAMS Innovation Fund for Medical Sciences (CIFMS 2018-I2M-1-003).

\section{ACKNOWLEDGMENTS}

We thank Hui Wang and Xuefu You for the kind gift of the E. coli clinical isolates.

\section{SUPPLEMENTARY MATERIAL}

The Supplementary Material for this article can be found online at: https://www.frontiersin.org/articles/10.3389/fmicb. 2021.676414/full\#supplementary-material

Cannatelli, A., Principato, S., Colavecchio, O. L., Pallecchi, L., and Rossolini, G. M. (2018). Synergistic Activity of Colistin in Combination With Resveratrol Against Colistin-Resistant Gram-Negative Pathogens. Front. Microb. 9:1808. doi: $10.3389 /$ fmicb.2018.01808

Capparelli, E. V., Bricker-Ford, R., Rogers, M. J., McKerrow, J. H., and Reed, S. L. (2017). Phase I Clinical Trial Results of Auranofin, a Novel Antiparasitic Agent. Antimicrob. Agents Chemother. 61:1. doi: 10.1128/aac.01947-16

Debnath, A., Parsonage, D., Andrade, R. M., He, C., Cobo, E. R., Hirata, K., et al. (2012). A high-throughput drug screen for Entamoeba histolytica identifies a new lead and target. Nat. Med. 18, 956-960. doi: 10.1038/nm.2758

Doern, C. D. (2014). When does 2 plus 2 equal 5? A review of antimicrobial synergy testing. J. Clin. Microb. 52, 4124-4128. doi: 10.1128/JCM.01 121-14

Falagas, M. E., Voulgaris, G. L., Tryfinopoulou, K., Giakkoupi, P., Kyriakidou, M., Vatopoulos, A., et al. (2019). Synergistic activity of colistin with azidothymidine against colistin-resistant Klebsiella pneumoniae clinical isolates collected from inpatients in Greek hospitals. Internat. J. Antimicrob. Agents 53, 855-858. doi: 10.1016/j.ijantimicag.2019.02.021

Flamm, R. K., Rhomberg, P. R., Lindley, J. M., Sweeney, K., Ellis-Grosse, E. J., and Shortridge, D. (2019). Evaluation of the Bactericidal Activity of Fosfomycin in Combination with Selected Antimicrobial Comparison Agents Tested against Gram-Negative Bacterial Strains by Using Time-Kill Curves. Antimicrob. Agents Chemother. 63:5. doi: 10.1128/AAC.02549-18 
Harbut, M. B., Vilchèze, C., Luo, X., Hensler, M. E., Guo, H., Yang, B., et al. (2015). Auranofin exerts broad-spectrum bactericidal activities by targeting thiol-redox homeostasis. Proc. Natl Acad. Sci. U S A 112, 4453-4458. doi: 10.1073/pnas. 1504022112

Hu, Y., Liu, Y., and Coates, A. (2019). Azidothymidine Produces Synergistic Activity in Combination with Colistin against AntibioticResistant Enterobacteriaceae. Antimicrob. Agents Chemother. 63:1. doi: 10.1128 /aac.01630-18

Hussein, M., Schneider-Futschik, E. K., Paulin, O. K. A., Allobawi, R., Crawford, S., Zhou, Q. T., et al. (2020). Effective Strategy Targeting Polymyxin-Resistant Gram-Negative Pathogens: Polymyxin B in Combination with the Selective Serotonin Reuptake Inhibitor Sertraline. ACS Infect. Dis. 6, 1436-1450. doi: 10.1021/acsinfecdis.0c00108

Clinical and Laboratory Standards Institute (2019). Performance standards for antimicrobial susceptibility testing. Wayne, PA: CLSI.

Kean, W. F., Hart, L., and Buchanan, W. W. (1997). Auranofin. Br. J. Rheumatol. 36, 560-572. doi: 10.1093/rheumatology/36.5.560

Koike, M., Iida, K., and Matsuo, T. (1969). Electron microscopic studies on mode of action of polymyxin. J. Bacteriol. 97, 448-452. doi: 10.1128/JB.97.1.448-452. 1969

Li, Y., Lin, X., Yao, X., Huang, Y., Liu, W., Ma, T., et al. (2018). Synergistic Antimicrobial Activity of Colistin in Combination with Rifampin and Azithromycin against Escherichia coli Producing MCR-1. Antimicrob. Agents Chemother. 62:12. doi: 10.1128/aac.01631-18

Lin, Y.-W., Yu, H. H., Zhao, J., Han, M.-L., Zhu, Y., Akter, J., et al. (2018). Polymyxin B in Combination with Enrofloxacin Exerts Synergistic Killing against Extensively Drug-Resistant Pseudomonas aeruginosa. Antimicrob. Agents Chemother 2:6. doi: 10.1128/AAC.00028-18

Liu, Y.-Y., Wang, Y., Walsh, T. R., Yi, L.-X., Zhang, R., Spencer, J., et al. (2016). Emergence of plasmid-mediated colistin resistance mechanism MCR-1 in animals and human beings in China: a microbiological and molecular biological study. Lancet. Infect.Dis. 16, 161-168. doi: 10.1016/S1473-3099(15)00424-7

Odds, F. C. (2003). Synergy, antagonism, and what the chequerboard puts between them. J. Antimicrob. Chemother. 52:1. doi: 10.1093/jac/dkg301

Olaitan, A. O., Morand, S., and Rolain, J.-M. (2014). Mechanisms of polymyxin resistance: acquired and intrinsic resistance in bacteria. Front. Microb. 5:643. doi: $10.3389 /$ fmicb. 2014.00643

Roder, C., and Thomson, M. J. (2015). Auranofin: repurposing an old drug for a golden new age. Drugs ReD 15, 13-20. doi: 10.1007/s40268-015-0083-y

Schneider, E. K., Azad, M. A. K., Han, M.-L., Tony Zhou, Q., Wang, J., Huang, J. X., et al. (2016). An "Unlikely" Pair: The Antimicrobial Synergy of Polymyxin B in Combination with the Cystic Fibrosis Transmembrane Conductance Regulator Drugs KALYDECO and ORKAMBI. ACS Infect. Dis. 2, 478-488. doi: 10.1021/ acsinfecdis.6b00035

Suarez-Almazor, M. E., Spooner, C. H., Belseck, E., and Shea, B. (2000). Auranofin versus placebo in rheumatoid arthritis. Cochrane Database Syst. Rev. 2:CD002048. doi: 10.1002/14651858.CD002048

Sun, H., Zhang, Q., Wang, R., Wang, H., Wong, Y.-T., Wang, M., et al. (2020). Resensitizing carbapenem- and colistin-resistant bacteria to antibiotics using auranofin. Nat. Commun. 11:5263. doi: 10.1038/s41467-020-18939-y

Sun, W., Weingarten, R. A., Xu, M., Southall, N., Dai, S., Shinn, P., et al. (2016). Rapid antimicrobial susceptibility test for identification of new therapeutics and drug combinations against multidrug-resistant bacteria. Emerg. Microb. Infect. 5:e116. doi: 10.1038/emi.2016.123

Thangamani, S., Mohammad, H., Abushahba, M. F. N., Sobreira, T. J. P., Hedrick, V. E., Paul, L. N., et al. (2016). Antibacterial activity and mechanism of action of auranofin against multi-drug resistant bacterial pathogens. Sci. Rep. 6:22571. doi: $10.1038 /$ srep 22571

Tharmalingam, N., Ribeiro, N. Q., da Silva, D. L., Naik, M. T., Cruz, L. I., Kim, W., et al. (2019). Auranofin is an effective agent against clinical isolates of Staphylococcus aureus. Future Med. Chem. 11, 1417-1425. doi: 10.4155/fmc2018-0544

Torres, N. S., Montelongo-Jauregui, D., Abercrombie, J. J., Srinivasan, A., LopezRibot, J. L., Ramasubramanian, A. K., et al. (2018). Antimicrobial and Antibiofilm Activity of Synergistic Combinations of a Commercially Available Small Compound Library With Colistin Against Pseudomonas aeruginosa. Front. Microb. 9:2541. doi: 10.3389/fmicb.2018.02541

Tran, T. B., Wang, J., Doi, Y., Velkov, T., Bergen, P. J., and Li, J. (2018). Novel Polymyxin Combination With Antineoplastic Mitotane Improved the Bacterial Killing Against Polymyxin-Resistant Multidrug-Resistant GramNegative Pathogens. Front. Microb. 9:721. doi: 10.3389/fmicb.2018.00721

Velkov, T., Thompson, P. E., Nation, R. L., and Li, J. (2010). Structure-activity relationships of polymyxin antibiotics. J. Med. Chem. 53, 1898-1916. doi: 10. 1021/jm900999h

Wang, R., van Dorp, L., Shaw, L. P., Bradley, P., Wang, Q., Wang, X., et al. (2018). The global distribution and spread of the mobilized colistin resistance gene mcr-1. Nat. Commun. 9:1179. doi: 10.1038/s41467-018-03205-Z

Wang, X., Wang, Y., Zhou, Y., Li, J., Yin, W., Wang, S., et al. (2018). Emergence of a novel mobile colistin resistance gene, mcr-8, in NDM-producing Klebsiella pneumoniae. Emerg. Microb. Infect. 7:122. doi: 10.1038/s41426-0180124-Z

Wang, Y.-M., Kong, L.-C., Liu, J., and Ma, H.-X. (2018). Synergistic effect of eugenol with Colistin against clinical isolated Colistin-resistant strains. Antimicrob. Resist. Infect. Control 7:17. doi: 10.1186/s13756-018-0303-7

Wang, Y., Tian, G.-B., Zhang, R., Shen, Y., Tyrrell, J. M., Huang, X., et al. (2017). Prevalence, risk factors, outcomes, and molecular epidemiology of mcr1-positive Enterobacteriaceae in patients and healthy adults from China: an epidemiological and clinical study. Lancet. Infect. Dis. 17, 390-399. doi: 10.1016/ S1473-3099(16)30527-8

Zhou, Y., Wang, T., Guo, Y., Liu, S., Wang, J., Shen, Y., et al. (2018). / Activity of Potential MCR-1 Inhibitor in Combination With Colistin Againsts -1-Positive. Front. Microb. 9:1615. doi: 10.3389/fmicb.2018.01615

Conflict of Interest: The authors declare that the research was conducted in the absence of any commercial or financial relationships that could be construed as a potential conflict of interest.

Copyright (c) 2021 Feng, Liu, Wang, Zhang, Sun, Li, Wang, Liu and Cao. This is an open-access article distributed under the terms of the Creative Commons Attribution License (CC BY). The use, distribution or reproduction in other forums is permitted, provided the original author(s) and the copyright owner(s) are credited and that the original publication in this journal is cited, in accordance with accepted academic practice. No use, distribution or reproduction is permitted which does not comply with these terms. 